\title{
Wilderness medicine in southern Africa
}

\begin{abstract}
Adventure is a path. Real adventure - self-determined, selfmotivated, often risky - forces you to have first-hand encounters with the world. The world the way it is, not the way you imagine it. Your body will collide with the earth and you will bear witness. In this way you will be compelled to grapple with the limitless kindness and bottomless cruelty of mankind - and perhaps realize that you yourself are capable of both. This will change you. Nothing will ever again be black-and-white. (The Ghost Road - Mark Jenkins)
\end{abstract}

As the cradle of humankind, southern Africa is considered by many to be the origin of the peripatetic human soul. From Africa, our species has explored and expanded to settle every continent and environment on the planet. The tip of the continent has long been associated with the ebb and flow of indigenous cultures, and also the first truly global explorers, offering safe harbours and supplies to the likes of Dias, Darwin, Drake and Da Gama. It was the ultimate point of departure for expeditions of the epic eras of Antarctic exploration, serving Cook and Scott, leading to South Africa (SA) becoming an original signatory member of the Antarctic Treaty. The natural beauty and wealth of wilderness environments to be found in SA have kindled a nation of outdoor enthusiasts, adventurers, sportsmen and women, and a strong ethos of exploration. Today, South Africans continue to drive exploration around the globe in the name of science, the environment and other good causes.

Where adventurers have fared, they have been forced to have first-hand encounters with the world ... collide with the earth ... and bear witness. The inherent risks of adventure and exploration have called for medicine to follow the path of man into the wilderness, providing care far from civilisation, and learning from human physiology under duress. Initially the role of the enthusiastic amateur physician-adventurer, this has led to the development of the field of wilderness and expedition (or extreme) medicine. While the discipline has become well established in parts of the world (particularly Europe and North America), Africa has traditionally been seen as the site of expeditions, rather than a role-player in the field. However, the practical experience, breadth and depth of knowledge and skill of SA medical practitioners should not be discounted. Although glamorously exotic destinations such as the Poles and Himalayas are more traditionally associated with high-profile expeditions, more aspiring summiteers die each year on 5895 metre-high Mount Kilimanjaro than 8848 metre-high Mount Everest. The diversity of African wilderness environments and outdoor lifestyle culture places our medics in an ideal position to become leaders in the field, and efforts to provide more formal training and support for wilderness medicine in SA are underway.

Wilderness and expedition medicine draws from a wide range of medical fields, yet defies easy classification. Traditionally, the type of wilderness environment has been used to group the knowledge and skills required. For instance, mountaineering expeditions require knowledge of high altitude physiology and illness, exposure injuries, and trauma care, whereas jungle expeditions require an understanding of tropical illnesses, envenomation and the challenging care of minor injuries. Polar, marine, desert and disaster environments all carry further special considerations. There are areas of intersection and overlap with many medical fields, beginning with general practice and emergency medicine, but also spanning sports medicine, tropical hygiene and infectious disease, aviation and maritime medicine, and occupational health. Medical professionals are often drawn into wilderness medicine through a particular recreational interest in one field (e.g. dive medicine), but are united by a fascination for the insights brought by the interaction of adverse environments and human physiology, and the challenges of treating the effects of physiological derangement or injuries occurring in the environments themselves. The continual increase in recreational use of the wilderness by an ever-widening spectrum of South Africans is, unfortunately, parallelled by an increasing need for high-quality care in environments separated from normal medicine by extremes of distance, environment and terrain (Fig 1).

In the spirit of growing the discipline in southern Africa, local enthusiasts and experts in the fields present this series of CME articles on wilderness medicine topics, with a focus on their relevance in the region. In this edition, the scope stretches from ocean to mountain, discussing the risks and management of high-altitude illnesses, ${ }^{[1]}$ an update on drowning in SA, ${ }^{[2]}$ and the management of cold-exposure injuries. ${ }^{[3]} \mathrm{A}$ further edition in the series will address expedition medicine, psychological and human factors, and heat-related illness. Finally, we introduce the fledgling Wilderness and Expedition Medicine Society of Southern Africa, formed to create a home and hub for interested practitioners around the country. It is our fervent hope that these efforts will enable a new generation of African wilderness medics to have first-hand encounters with the world, and be changed for the better.

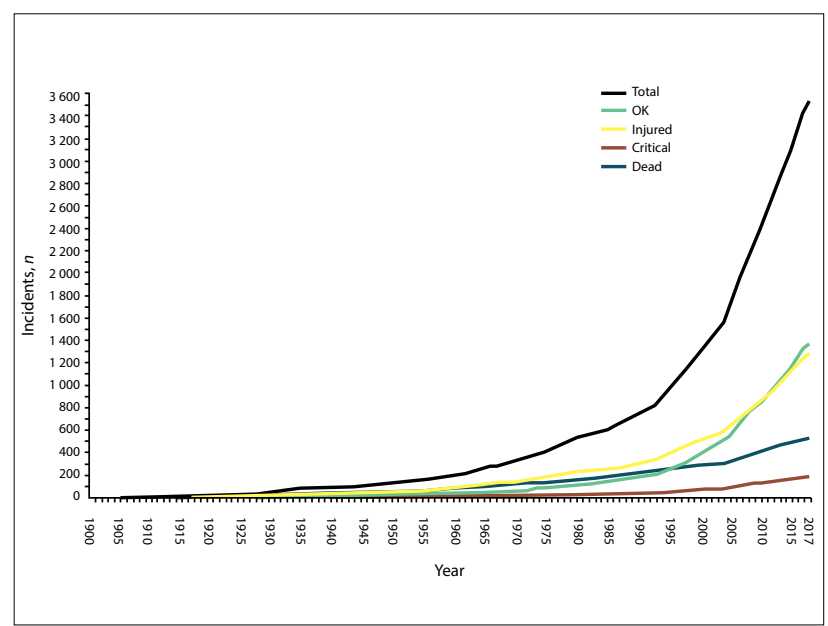

Fig. 1. Cumulative wilderness rescue statistics from 1900 to early 2017, courtesy of the SA Mountain Accidents Database. 


\section{Ross Hofmeyr}

Department of Anaesthesia and Perioperative Medicine, Faculty of Health Sciences, University of Cape Town; and WildMedix, Cape Town, South Africa ross.hofmeyr@uct.ac.za

\section{Jay Matthew}

Department of Emergency Medicine, Faculty of Health Sciences, University of KwaZulu-Natal, Durban; and Lifesaving South Africa, Durban, South Africa
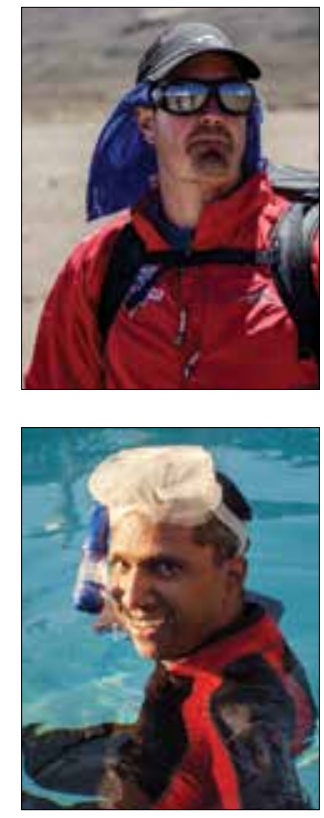

\section{Rik De Decker}

Department of Paediatrics and Child Health, Faculty of Health Sciences, University of Cape Town, and Red Cross War Memorial Children's Hospital, Cape Town, South Africa

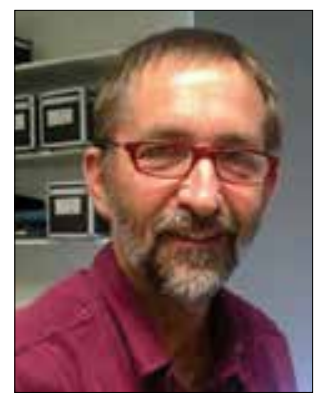

1. Hofmeyr R, Tölken G, De Decker R. Acute high-altitude illness. S Afr Med J 2017;107(7):556-561. https://doi.org/10.7196/SAMJ.2017.v107i7.12612

2. Matthew J, Robertson C, Hofmeyr R. Update on drowning. S Afr Med J 2017;107(7):562-565. https:// doi.org/10.7196/SAMJ.2017.v107i7.12609

3. Hofmeyr R. Wilderness cold-exposure injuries: An African Perspective. S Afr Med J 2017;107(7):566570. https://doi.org/10.7196/SAMJ.2017.v107i7.12610

S Afr Med J 2017;107(7):554-555. DOI:10.7196/SAMJ.2017.v107i7.12608 